\title{
Performance Improvement of Base Fluid Heat Transfer Medium Using Nano Fluid Particles
}

\author{
T. Sathish
}

\author{
Department of Mechanical Engineering, Saveetha School of Engineering, SIMATS, Chennai, Tamil Nadu - 602 105, India
}

Corresponding Author Email: sathish.sailer@gmail.com

\begin{abstract}
Base fluids like water, ethylene glycolandengineoilare conventionally used as a heat transfer medium. The performance of heat transferred is improved in the conventional fluids with the addition of Nano particles. Hence, this paper considers the forced conventional flow problem over the base fluid within a uniform heated tube placed on a wall. The analysis of heattransferco-efficientis done through a constant Reynoldsnumberfor both Nano and base fluid with a simulation tool. Further, a comparative analysis is carried out with heat transfer coefficient over the base and various Nano fluids. It is seen that the Nano fluids has a better performance due to its better thermal characteristics under standard conditions.
\end{abstract}

Keywords: heat transfer coefficient, CFX simulation, ansys simulation, nano fluid and base fluid Received: July-20-2020, Accepted: October-26-2020, https://doi.org/10.14447/jnmes.v23i4.a03

\section{INTRODUCTION}

The Nano fluids contains fluid with Nanometer-sized particles [1], which has good stability, increased thermal conductivity even with small suspended Nanoparticles and heat transferring performance. The Nano fluidmaterials vary based on metal oxides, chemically stable metals, metal carbides, oxide ceramics etc. However, most literatures focus on single-particle based Nano fluids to test the thermal characteristics [2]. The thermal fluids like oil, water and ethylene glycol plays a major role in cooling process in many power applications. However, these fluid contains poor thermal properties and the properties of heat transfer rate is affected. Hence, to heat transfer coefficient is improved with the help of extended surface channels, surface vibration and fluid injection. The use of new technology is being an enhancing factor to improve the thermal properties of such substance [3].

The main advantage of Nano fluids include ling term stability, superior thermal conductivity and reduced drop in pressure, when compared with $\mathrm{mm}$ and $\mu \mathrm{m}$ sized particle. The materials like metal carbides, oxide ceramics, metals, nitrides, nonmetals, carbon Nanotubes and Nanoparticles is used to form the Nano fluid. A synthesis optimization is used to make the suspensions of Nanoparticle to be stable inside the base fluid.

In order to improve the thermal properties, several combinations of base fluid with Nano particle is possible. Also, the Nano fluids are produced using a single step or two step method, where the former one uses formation and dispersion of Nanoparticles within the base fluid, using several experimental procedures [4]. Hence, the use of storage, drying, transportation and dispersion is avoided with such method.

The solid substance possesses better thermal conductivities than other substances. Most researches concentrated on the thermal properties associated with the suspension of solid particles in conventional fluids. Such dispersed particles vary from $\mathrm{mm}$ to $\mu \mathrm{m}$ size over the base fluid and this leads to change in thermo-physical properties of such fluid and that results in enhancing the heat transfer ratio. The main disadvantage of such $\mathrm{mm}$ to $\mu \mathrm{m}$ sized particle leads to poor stability and suspension and this results in channel clogging. However, recent advances with the help of new materials improves the thermal properties of the fluid than the conventional fluids [5]. From conventional studies, it is seen than the thermal conductivity of the Nano fluids is found higher than base fluid [6-8].

Out of which, water is used as a base fluid, where a rod and spherical shaped $\mathrm{TiO}_{2}[9]$ Nanoparticles are dispersed in water. The use of aqueous/non-aqueous solution as base fluid is used with metal oxide particles of varying shapes and concentration [10]. The water is further used with $\mathrm{Al}_{2} \mathrm{O}_{3}[11,12], \mathrm{Fe}_{3} \mathrm{O}_{4}$ [13], $\mathrm{NH}_{3}$ [14], $\mathrm{Al}_{2} \mathrm{O}_{3}$ and $\mathrm{TiO}_{2}$ [15], $\mathrm{MgO}$ [16], $\mathrm{COOH}$ [17], to measure the $\mathrm{pH}$ effect with varying thermal conductivity. With different fractional weights, the copper based water base fluid is used to measure the $\mathrm{pH}$ rate with sodium dodecyl benzene sulfonate surfactant [18]. Also, water is used with carbon Nano tubes and $\mathrm{TiO}_{2}$ [19] with carbon Nano tubes measures the time and temperature [20] variations and Nanodiamond particle with deionized fluid measures varying loads [21]. Nanoparticlesgraphene [22], Nanoshell [23], graphene oxide Nanosheets [24] and Nano diamond [25] with water as base fluids.

The use of Ethylene Glycol as base fluid is experimented in several researches, which includes: synthetic EG multiwalled carbon Nanotubes [26], ZnO-EG Nano fluids [27, 28], Al-Zn with EG Nanoparticles [29], graphene Nanosheets with EG [30], Aluminum nitride with EG Nanoparticle [31], $\mathrm{Al}_{2} \mathrm{Cu}$ and $\mathrm{Ag}_{2} \mathrm{Al}$ with EG Nanoparticles [32], $\mathrm{Al}_{2} \mathrm{O}_{3}, \mathrm{CuO}, \mathrm{ZnO}$ with EG [33], $\mathrm{TiO}_{2}$ Nanoparticles with EG [34], $\mathrm{CuO}$ Nanoparticles [35], Cu Nanoparticles [36], $\alpha-S i C$ Nano fluids [37], spherical $\mathrm{ZnO}$ Nanoparticles [38], $\mathrm{Al}_{2} \mathrm{O}_{3}$ with $\mathrm{EG}$ and water [39, 40].

The increased techniques to improve the heat transfer coefficients suffer majorly the important strategy required to 
achieve effective heat transfer over conventional base fluid. Hence, the heat transfer is intensified with the suspension of Nanoparticles with the use of base fluid. In the proposed work, we considered the problem of improving the heat transfer coefficient by the use of Nano fluids. Here, base fluids like engine oil, ethylene glycol and water and the Nanoparticles of gold, copper, aluminum and silver is used. Empirical relations are used to calculate the properties of the Nano fluids. Further, the thermo-physical properties of ethylene glycol, water, copper, engine oil, gold, aluminum and silver is calculated to find the Nano fluid properties. The heat transfer rate of the Nano and base fluid is analysed with the use of CFX simulation tool under a constant Reynolds number. Finally, comparative analysis is carried between the thermal conductivity models namely, Maxwell, Hamilton and Crosser, and Davis models [41, 42].

The outline of the paper is mentioned as follows: Section 2 provides the proposed model to analyse the flow rate in base and Nano fluid analysis. Section 3 provides the experimental method for finding the thermo-physical properties. Section 4 provides the evaluation on different combinations of Nano fluids with base fluid. Section 5 concludes the paper.

\section{METHODS}

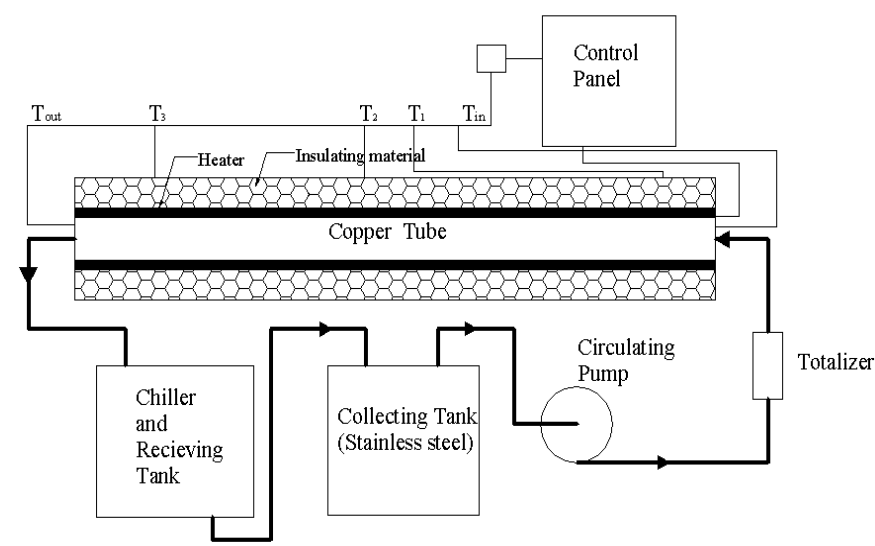

Figure 1. Experimental Setup

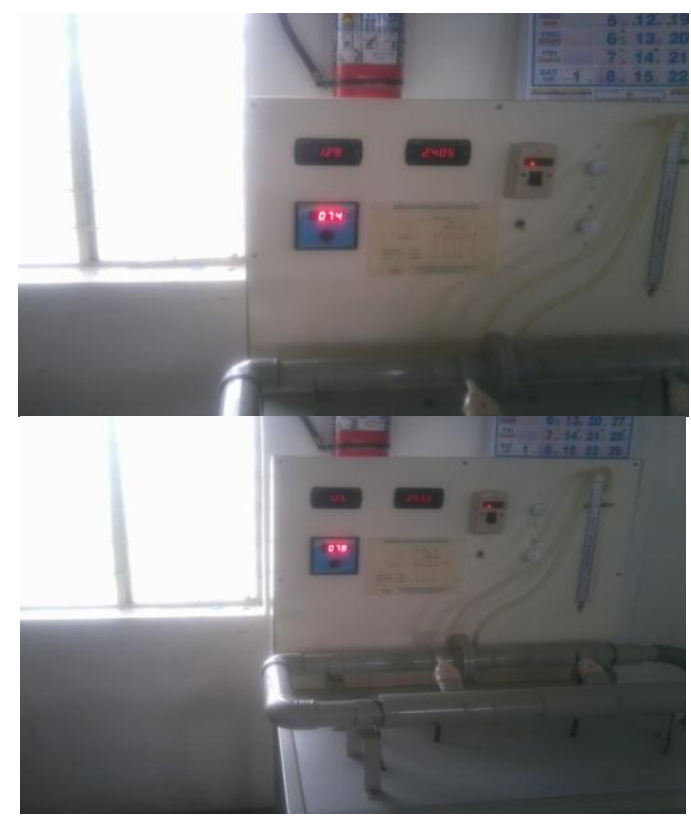

Figure 2. Geometry of Tube
The experimental setup of the proposed method is shown in Figure 1. The system has alooping pipeline flow structure, pumping and copper tube of length 1 mand diameter $60 \mathrm{~mm}$ and are servoir tank. Further, the tube is insulated with $25 \mathrm{~mm}$ glass made of fiber and heat electrically at $5000 \mathrm{~W} / \mathrm{m}^{2}$. The tube is coated with nickel chrome wire, which is connected with a 300W DC supply. The tube is installed with four k type tube, where two of which is placed at two end. This is used to measure the temperature of the Nano fluid.

The numerical simulation uses single phase method to find the values of convective heat transfer. Here, the geometry of the tube is shown in Figure 2. The tube allows the Nano fluidto enter at a uniform velocity rate $\left(U_{1}\right)$ and the rate of temperature $\left(T_{1}\right)$. The condition of full development is assumed at the tube end. Based on the tube's centerline, the assumptions are made symmetrically on thermal and velocity fields. The local heat transfer coefficient rate is $h_{\text {local }}$ :

$$
h_{\text {local }}=\frac{\left(T_{\text {local }}-T_{\text {input }}\right) \rho C p u D}{4 \pi\left(T_{w}-T_{\text {input }}\right)}
$$

The averagerate of heattransferco-efficient is given as $h_{\text {ave }}$ :

$$
h_{\text {ave }}=\frac{1}{L \int_{0}^{L} h(z) d z}
$$

The Average Nusselt number is then given by:

$$
N u_{a v e}=\frac{h_{a v e} D}{K_{a f}}
$$

Heat transfer(Q)

$$
Q=\sqrt{\frac{C_{d} a_{1} a_{2}\left(2 g h_{a}\right)}{\left(a_{1}^{2}-a_{2}^{2}\right)}}
$$

\section{ESTIMATION THERMO-PHYSICAL PROPERTIES}

The measuring the thermal conductivity of the Nano fluid use heat transfer coefficient, both in forced and natural fluid flow. The main limitation involves thermal characteristics of the Nanoparticles without changing the phase. The proposed model uses two phase systems for finding the heat transfer ratio of particles in the Nano fluid. Here, the coefficient of heat transfer rate depends on thermal conductivity, density, specific heat ratio and dynamic viscosity of particles in the Nano fluid. The properties of particles in the Nano fluid at normal temperature is shown in Table 1 and the properties of base fluid at normal temperature is shown in Table 2.

\subsection{Density Estimation}

The Nanofluiddensity is calculatedwith massbalance, which is given as:

$$
\rho_{n f}=\left(1-\varphi_{S}\right) \rho_{f+} \varphi_{S} \rho_{p}
$$

The particles present in the Nano fluid has a volume fraction $<1 \%$ and the changeof $<5 \%$ fluiddensityis thus expected. Further, the density for $1 \%$ particles with copper mix and 
water as base fluid, the Nano fluid density is found to be $1075.865 \mathrm{Kg} / \mathrm{m}^{3}$. From Eq. (5), the Nano fluid density for different $\%$ mixes i.e. $1,3,5, \ldots, 25$, various combinations are calculated, which is shown in Table.3. The different mixture of Nano fluids include: copper as base fluid with water as Nano fluid, gold as base fluid with water as Nano fluid, silver as base fluid with water as Nano fluid, aluminum as base fluid with water as Nano fluid, copper as base fluid with ethyleneglycol as Nano fluid, gold as base fluid and ethyleneglycol as Nano fluid, silver as base fluid with ethyleneglycol as Nano fluid, aluminum as base fluid with ethyleneglycol as Nano fluid, copper as base fluid with engine oil as Nano fluid, gold as base fluid with engine oil as Nano fluid, silver as base fluid with engine oil as Nano fluid and finally aluminum as base fluid with engine oil as Nano fluid. The density of these base fluid and Nano fluid mixtures are shown in Table 3.

\subsection{Specific heat estimation}

The estimation of specific heat of Nano fluids with mass balance is calculated as:

$$
\left(1-\varphi_{S}\right) \rho_{f} C_{f}+\varphi_{S} \rho_{p} C_{P}
$$

where, $C_{n f}=\rho_{n f}$, a small reduction over the Nano particle's specific heat while the dispersion of solid particle in liquid is estimated using following calculations. Further, the specific heat for $1 \%$ particles with aluminum oxide mix and water as base fluid, the Nano fluid density is found to be $3863.981 \mathrm{~J} / \mathrm{KgK}$. The specific heat for other Nano-fluid mixes for different percentage is shown in Table 4.

\subsection{Velocity of Nano Fluid}

The input parameter for the simulation model is the velocity of Nano fluid possessing its own materialistic properties. Initially, the co-efficient for heat transfer rate is estimated for base and Nano fluid using constant Reynold number, where $R e$ $=u d \rho / \mu=2000$. The velocity of the water is found as $0.29 \mathrm{~m} / \mathrm{s}$, and for ethylene glycol $=4.7 \mathrm{~m} / \mathrm{s}$ and engine oil is $183 \mathrm{~m} / \mathrm{s}$. Further, the velocity of different Nano-fluid mixes for different percentage is shown in Table 5.

Table 1. Nano particles Properties

\begin{tabular}{ccccc}
\hline Property & \multicolumn{4}{c}{ Copper Gold SilverAluminum } \\
\hline Thermal conductivity $(\mathrm{Ks}) \mathrm{W} / \mathrm{mK}$ & 401 & 317 & 429 & 237 \\
Density $\left(\rho_{\mathrm{p}}\right) \mathrm{kg} / \mathrm{m}^{3}$ & 8933 & 1930010500 & 2702 \\
Specific heat $(\mathrm{CP}) \mathrm{J} / \mathrm{kg} \mathrm{K}$ & 385 & 129 & 235 & 903 \\
\hline
\end{tabular}

Table 2. Base Fluid Properties

\begin{tabular}{|c|c|c|c|}
\hline Property & Water & E. glyco & ngine Oil \\
\hline Thermal conductivity $\left(\mathrm{K}_{\mathrm{f}}\right) \mathrm{W} / \mathrm{mK}$ & 0.613 & 0.252 & 0.145 \\
\hline Density $\left(\rho_{\mathrm{f}}\right) \mathrm{kg} / \mathrm{m}^{3}$ & 996.5 & 1114.4 & 884.1 \\
\hline Specific heat $\left(\mathrm{C}_{\mathrm{f}}\right) \mathrm{J} / \mathrm{kgK}$ & 4179 & 2415 & 1909 \\
\hline Dynamic viscosity $\left(\mu_{\mathrm{f}}\right) \mathrm{Kg} / \mathrm{ms}$ & 0.00085 & 0.0157 & 0.486 \\
\hline
\end{tabular}

Table 3. Various Nano fluid Density (in $\mathrm{Kg} / \mathrm{m}^{3}$ )with different base fluid combinations

\begin{tabular}{|c|c|c|c|c|c|c|c|c|c|c|c|}
\hline $\begin{array}{c}\text { Volume } \\
\text { fraction } \\
(\%)\end{array}$ & $\begin{array}{l}\text { copper- } \\
\text { water }\end{array}$ & $\begin{array}{l}\text { silver- } \\
\text { water }\end{array}$ & $\begin{array}{c}\text { aluminum } \\
\text { - } \\
\text { water }\end{array}$ & $\begin{array}{c}\text { copper- } \\
\text { Ethylene } \\
\text { glycol }\end{array}$ & $\begin{array}{c}\text { gold- } \\
\text { Ethylene } \\
\text { glycol }\end{array}$ & $\begin{array}{l}\text { silver- } \\
\text { Ethylene } \\
\text { glycol }\end{array}$ & $\begin{array}{c}\text { aluminum- } \\
\text { Ethylene } \\
\text { glycol }\end{array}$ & $\begin{array}{c}\text { copper- } \\
\text { Engine } \\
\text { Oil }\end{array}$ & $\begin{array}{c}\text { gold- } \\
\text { Engine } \\
\text { Oil }\end{array}$ & $\begin{array}{c}\text { silver- } \\
\text { Engine } \\
\text { Oil }\end{array}$ & $\begin{array}{l}\text { aluminum- } \\
\text { Engine Oil }\end{array}$ \\
\hline 1 & & $5 \overline{1091.535}$ & 1013.555 & 1192.586 & 1296.256 & 1208.256 & 1130.276 & 964.589 & 1068.259 & 980.259 & 902.279 \\
\hline 3 & & 605 & & & & & & & & & \\
\hline 5 & & 5147 & 1081.775 & & & 158 & & & & & \\
\hline 7 & & 745 & .885 & 166 & 2387. & & & & & & \\
\hline 9 & 1710. & 51851.815 & 1149.995 & 181 & 2751.104 & & & & & & \\
\hline 11 & 1869.51 & 3009.8852041 .885 & & 1974.446 & 3114.816 & 214 & & 1769. & 290 & & .069 \\
\hline 13 & 2028.24 & 3375.9552231 .955 & 1218.215 & 2130.818 & 3478.528 & 2334. & & 1930. & 327 & 213 & .427 \\
\hline 15 & 2186.97 & 3742.0252422 .025 & 1252.325 & 2287.19 & 3842.24 & 2522.24 & & 2091. & 364 & 2326 & 6.785 \\
\hline 17 & 2345.705 & 4108.0952612 .095 & 1286.435 & 2443.562 & 4205.952 & 2709.952 & 1384.2 & 2252.413 & 4014.803 & 2518.803 & 1193.143 \\
\hline 19 & 2504.43 & 4474.1652802 .165 & 1320.545 & 2599.934 & 4569.664 & 2897.664 & 1416.0 & 2413.391 & 4383.121 & 2711.121 & 1229.501 \\
\hline 21 & 2663.16 & 4840.2352992 .235 & 1354.655 & 2756.306 & & & & & & & \\
\hline 23 & 2821.89 & 5206.3053182 .305 & & 2912 & $529^{\circ}$ & & & & & & \\
\hline 25 & 2980.625 & 5572.3753372 .375 & 1422.875 & 3069.05 & 5660.8 & 3460.8 & 1511.3 & 2896.325 & 5488.075 & 3288.075 & 1338.575 \\
\hline
\end{tabular}

Table 4. Nano fluid Specific Heat Capacity (inJ/KgK) with different base fluid combinations

\begin{tabular}{|c|c|c|c|c|c|c|c|c|c|c|c|c|}
\hline $\begin{array}{c}\text { Nano } \\
\text { particle } \%\end{array}$ & $\begin{array}{c}\text { copper- } \\
\text { Water }\end{array}$ & $\begin{array}{l}\text { gold- } \\
\text { Water }\end{array}$ & $\begin{array}{l}\text { silver- } \\
\text { Water }\end{array}$ & $\begin{array}{c}\text { Aluminum } \\
\text {-Water }\end{array}$ & $\begin{array}{c}\text { copper- } \\
\text { Ethylene } \\
\text { glycol }\end{array}$ & $\begin{array}{c}\text { gold- } \\
\text { Ethylene } \\
\text { glycol }\end{array}$ & $\begin{array}{c}\text { silver- } \\
\text { Ethylene } \\
\text { glycol }\end{array}$ & $\begin{array}{l}\text { aluminum- } \\
\text { Ethylene } \\
\text { glycol }\end{array}$ & $\begin{array}{c}\text { copper- } \\
\text { Engine } \\
\text { Oil }\end{array}$ & $\begin{array}{c}\text { gold- } \\
\text { Engine } \\
\text { Oil }\end{array}$ & $\begin{array}{c}\text { silver- } \\
\text { Engine } \\
\text { Oil }\end{array}$ & $\begin{array}{l}\text { aluminum- } \\
\text { Engine Oil }\end{array}$ \\
\hline 1 & 3863.98 & 25162 & 3799.608 & 4091.666 & 2262.944 & 2074.637 & 2225.553 & 2378.855 & 1767.863 & 1587.411 & 1729.69 & 1878.874 \\
\hline 3 & & & & 3925 & 2011.711 & 1617.639 & 1923.083 & 27 & & & 1459.298 & \\
\hline 5 & & & & & & 912 & & & & 103 & 104 & .604 \\
\hline 7 & 2650.42 & & & & & 1121.377 & & & & & & 1720.861 \\
\hline 9 & 2396.0 & & 2166.337 & 3486.251 & & 971.6587 & 1363.448 & & & 692.4656 & 1004.798 & 5.501 \\
\hline 11 & & & & 3356.697 & & 856.9054 & 1242 & & & 278 & & \\
\hline 13 & & & & & & & & & & & & \\
\hline 15 & 1854.433 & 1045.737 & 1614.287 & 3118.762 & 1225.725 & 692.5751 & 1053.71 & .916 & .5968 & 495.8309 & 775.7238 & 1556.53 \\
\hline 17 & 1722.763 & 944.3986 & 1483.83 & 3009.257 & 1153.408 & 631.7257 & 979.0705 & & 881.4968 & .3383 & 722.6865 & 1521.707 \\
\hline 19 & & & & & & 580.5627 & & & & 41 & 1738 & \\
\hline 21 & 1506.511 & 787.70 & 1272.637 & 2806.792 & 1033.391 & 536.9437 & 857.0375 & 1822.417 & 798. & 390 & 904 & .062 \\
\hline 23 & 1416.631 & 725.8888 & 1185.962 & 2713.019 & 983.0471 & 499.3146 & 806.519 & 1779.909 & 764.2841 & 365.6807 & 603.1126 & 1428.904 \\
\hline 25 & 1336.3246 & 672.1919 & 1109.057 & 2623.742 & 937.8336 & 466.521 & 761.4806 & 1739.187 & 733.8995 & 344.0615 & 572.5798 & 1401.331 \\
\hline
\end{tabular}


Table 5. Velocity (in $\mathrm{m} / \mathrm{s}$ ) of Different Nano fluid mixtures

\begin{tabular}{|c|c|c|c|c|c|c|c|c|c|c|c|c|}
\hline $\begin{array}{c}\text { Nano } \\
\text { particle \% }\end{array}$ & $\begin{array}{l}\text { copper- } \\
\text { Water }\end{array}$ & $\begin{array}{l}\text { gold- } \\
\text { Water }\end{array}$ & $\begin{array}{l}\text { silver- } \\
\text { Water }\end{array}$ & $\begin{array}{c}\text { aluminum } \\
\text {-Water }\end{array}$ & $\begin{array}{l}\text { copper- } \\
\text { Ethylene } \\
\text { glycol }\end{array}$ & $\begin{array}{l}\text { gold- } \\
\text { Ethylene } \\
\text { glycol }\end{array}$ & $\begin{array}{c}\text { silver- } \\
\text { Ethylene } \\
\text { glycol }\end{array}$ & $\begin{array}{l}\text { aluminum- } \\
\text { Ethylene } \\
\text { glycol }\end{array}$ & $\begin{array}{c}\text { copper- } \\
\text { Engine } \\
\text { Oil }\end{array}$ & $\begin{array}{l}\text { gold- } \\
\text { Engine } \\
\text { Oil }\end{array}$ & $\begin{array}{c}\text { silver- } \\
\text { Engine } \\
\text { Oil }\end{array}$ & $\begin{array}{l}\text { aluminum- } \\
\text { Engine Oil }\end{array}$ \\
\hline 1 & 0.26490 & 0241621 & 0.2611 & 0.281188 & 4.388223 & 4.037268 & 4.331312 & & 167.9472 & 151.6486 & 165.2624 & \\
\hline 3 & & & & & & & & & & & & \\
\hline 5 & & & & & & & & & & & & \\
\hline 7 & 18267 & 012512 & 6 & 0.25 & & 92071 & 2.95 & & & & & \\
\hline 9 & & & & & & & & & & & & \\
\hline 11 & 52 & 0.09468 & & & & 142 & & & & & 564 & \\
\hline & & 0.08 & & & & & & & & & 784 & 877 \\
\hline 15 & 0.1303 & 0076 & 0.11767 & & & 2053 & & & & & 294 & \\
\hline 17 & & & & & & 4268 & & & & & & \\
\hline 19 & 0.113 & & & & & & & & & & & \\
\hline 21 & 0.107016 & 0.0588810 & 0.095247 & 0.210386 & 1.898676 & 1.060802 & 1.696174 & & 62.92804 & & 55.7959 & 127.9763 \\
\hline 23 & 0.10099 & 0.05474 & 0.089558 & 0.205218 & 1.796743 & 0.987964 & 1.598898 & & 59.22466 & & 52.32969 & 124.4032 \\
\hline 25 & 0.095618 & 0.051145 & 0.08451 & 0.200299 & 1.705197 & 0.924487 & 1.512174 & 3.462802 & 55.93295 & 29.51855 & 49.26895 & 121.0242 \\
\hline
\end{tabular}

\subsection{Viscosity of Nano fluids}

The Nano fluid viscosity is estimated using 2 phase mixtures,

$$
\mu=\mu_{0}\left(123 \varphi_{s}^{2}+7.3 \varphi_{S}+1\right)
$$

Further, the viscosity of $1 \%$ particles with copper mix and water as base fluid, the Nano fluid density is $0.000928 \mathrm{~kg} / \mathrm{ms}$. The combination of Nano fluid with water, ethylene glycol and engine oil is estimated and shown in Table 6.

\subsection{Nano fluid Thermal Conductivity}

TheNano fluid contains smaller particles with high thermal conductivity than the base fluid. The thermal conductivity depends entirely on particle volume fraction for its better enhancement. The thermal conductivity of the Nano fluids are estimated using three models, which depends entirely on the particle shape:

3.5.1. Maxwell Model

This model considers the spherical Nano particle and the relation is given by,

$$
K=K_{L} \frac{K_{S}+2 K_{L}+2\left(K_{S}-K_{L}\right) \phi_{S}}{K_{S}+2 K_{L}-\left(K_{S}-K_{L}\right) \phi_{S}}
$$

3.5.2. Hamilton and Crosser Model

This model considers the non-spherical Nanoparticle and the relation is given by,

$$
K=K_{L} \frac{K_{S}+(n-1) K_{L}-(n-1)\left(K_{L}-K_{S}\right) \phi_{S}}{K_{S}+(n-1) K_{f}-\left(K_{L}-K_{S}\right) \phi_{S}}
$$

where $n$ totally depends on the shape of particleand $\mathrm{K}_{\mathrm{S}} / \mathrm{K}_{\mathrm{L}}$, Also, $n$ is $3 / \psi \forall \mathrm{K}_{\mathrm{S}} / \mathrm{K}_{\mathrm{L}}>100$ and the value of $\mathrm{n}$ is 3forothercases

\subsubsection{Davis Model}

This model considers the tubularNanoparticle and the relation is given by,

$$
K=K_{L} \times 1+\frac{3\left(\frac{K_{S}}{K_{L}-1}\right)\left[\phi_{S}+f . \phi_{S}^{2}+O\left(\phi_{S}^{3}\right)\right]}{\left(\frac{K_{S}}{K_{L}+2}\right)-\left(\frac{K_{S}}{K_{L}-1}\right) \phi_{S}} \times K_{L}
$$

Table 6. Nano fluid Viscosity (in $\mathrm{kg} / \mathrm{ms}$ ) with water, ethylene glycol and engine oil

\begin{tabular}{cccc}
\hline \multirow{2}{*}{$\begin{array}{c}\text { Nanoparticle } \\
\text { \% }\end{array}$} & Nanoparticle-Water & Nanoparticle- Nanoparticle- \\
Ethyleneglycol & Engineoil \\
\hline 1 & 0.000928 & 0.017039 & 0.527456 \\
\hline 3 & 0.001137 & 0.020876 & 0.646234 \\
\hline 5 & 0.00143 & 0.026258 & 0.812835 \\
\hline 7 & 0.001807 & 0.033185 & 1.027258 \\
\hline 9 & 0.002269 & 0.041657 & 1.289504 \\
\hline 11 & 0.002814 & 0.051673 & 1.599572 \\
\hline 13 & 0.003444 & 0.063235 & 1.957462 \\
\hline 15 & 0.004157 & 0.076341 & 2.363175 \\
\hline 17 & 0.004955 & 0.090992 & 2.81671 \\
\hline 19 & 0.005837 & 0.107189 & 3.318068 \\
\hline 21 & 0.006803 & 0.12493 & 3.867248 \\
\hline 23 & 0.007854 & 0.144215 & 4.46425 \\
\hline 25 & 0.008988 & 0.165046 & 5.109075 \\
\hline
\end{tabular}

Table 7(a). Nano fluid thermal conductivity

\begin{tabular}{clcccccc}
\hline \multirow{2}{*}{$\begin{array}{c}\text { Nano } \\
\text { particle } \%\end{array}$} & \multicolumn{3}{c}{ Water \& Copper } & \multicolumn{2}{c}{ Ethylene glycol \& Copper } & \multicolumn{2}{c}{ Engine oil \& Copper } \\
\cline { 2 - 8 } & Maxwell & Hamilton and crosser & \multicolumn{1}{c}{ Davis } & MaxwellHamilton and crosser & Davis & MaxwellHamilton and crosser & Davis \\
\hline 1 & 0.63149 & 0.619042 & 0.6316770 .259622 & 0.25449 & 0.2596990 .149389 & 0.146434 & 0.149433 \\
\hline 3 & 0.669608 & 0.630775 & 0.6713570 .275336 & 0.259326 & 0.2760570 .158439 & 0.149219 & 0.158854 \\
\hline 5 & 0.709324 & 0.642063 & 0.7143810 .291711 & 0.263978 & 0.2937950 .167869 & 0.151898 & 0.169069 \\
\hline 7 & 0.750739 & 0.652931 & 0.7610560 .308788 & 0.268457 & 0.3130410 .177704 & 0.154476 & 0.180153 \\
\hline 9 & 0.793966 & 0.663402 & 0.8117180 .326615 & 0.272771 & 0.3339340 .187971 & 0.156961 & 0.192186 \\
\hline 11 & 0.839125 & 0.673497 & 0.8667350 .345241 & 0.276931 & 0.3566250 .198699 & 0.159355 & 0.205255 \\
\hline 13 & 0.88635 & 0.683237 & 0.9265050 .364721 & 0.280943 & 0.381280 .209919 & 0.161665 & 0.219456 \\
\hline 15 & 0.935785 & 0.692638 & 0.9914660 .385116 & 0.284816 & 0.4080790 .221667 & 0.163895 & 0.234892 \\
\hline 17 & 0.98759 & 0.70172 & 1.0620960 .406492 & 0.288556 & 0.4372210 .23398 & 0.166049 & 0.251678 \\
\hline 19 & 1.041938 & 0.710498 & 1.1389210 .428921 & 0.292172 & 0.4689230 .246901 & 0.16813 & 0.26994 \\
\hline 21 & 1.099022 & 0.718986 & 1.2225210 .452483 & 0.295667 & 0.5034260 .260474 & 0.170143 & 0.289816 \\
\hline 23 & 1.159054 & 0.7272 & 1.3135330 .477266 & 0.29905 & 0.5409940 .274752 & 0.17209 & 0.311459 \\
\hline 25 & 1.222269 & 0.735151 & 1.4126650 .503368 & 0.302324 & 0.581920 .289791 & 0.173975 & 0.335038 \\
\hline
\end{tabular}


Table 7(a). Nano fluid thermal conductivity (continued)

\begin{tabular}{cccccccc}
\hline \multirow{2}{*}{$\begin{array}{c}\text { Nano } \\
\text { particle \% }\end{array}$} & \multicolumn{3}{c}{ Water \& Gold } & \multicolumn{3}{c}{ Engine oil \& Gold } & \multicolumn{2}{c}{ Ethylene glycol \& Gold } \\
\cline { 2 - 8 } & Maxwell & Hamilton and crosser & Davis & MaxwellHamilton and crosser & Davis & MaxwellHamilton and crosser & Davis \\
\hline 1 & 0.631467 & 0.619035 & 0.6316540 .188879 & 0.146434 & 0.1494320 .259618 & 0.254489 & 0.259695 \\
\hline 3 & 0.669538 & 0.630754 & 0.6712850 .192767 & 0.149218 & 0.158850 .275324 & 0.259323 & 0.276045 \\
\hline 5 & 0.709201 & 0.64203 & 0.7142510 .19682 & 0.151896 & 0.1690620 .29169 & 0.263973 & 0.293773 \\
\hline 7 & 0.75056 & 0.652886 & 0.7608630 .201046 & 0.154474 & 0.1801420 .308758 & 0.268449 & 0.313009 \\
\hline 9 & 0.793725 & 0.663346 & 0.8114540 .205458 & 0.156957 & 0.1921710 .326574 & 0.272762 & 0.333889 \\
\hline 11 & 0.838817 & 0.673431 & 0.866390 .210068 & 0.159352 & 0.2052360 .345188 & 0.276919 & 0.356567 \\
\hline 13 & 0.885969 & 0.683161 & 0.9260690 .21489 & 0.161661 & 0.2194310 .364656 & 0.28093 & 0.381206 \\
\hline 15 & 0.935325 & 0.692554 & 0.9909260 .219938 & 0.16389 & 0.2348610 .385038 & 0.284801 & 0.407987 \\
\hline 17 & 0.987043 & 0.701628 & 1.061440 .225229 & 0.166044 & 0.2516410 .406399 & 0.288541 & 0.437109 \\
\hline 19 & 1.041297 & 0.710398 & 1.1381350 .230781 & 0.168125 & 0.2698960 .428812 & 0.292155 & 0.46879 \\
\hline 21 & 1.098278 & 0.71888 & 1.2215870 .236613 & 0.170137 & 0.2897640 .452357 & 0.295649 & 0.503267 \\
\hline 23 & 1.158196 & 0.727087 & 1.3124320 .242748 & 0.172084 & 0.3113970 .47712 & 0.299031 & 0.540807 \\
\hline 25 & 1.221286 & 0.735033 & 1.4113750 .249209 & 0.173968 & 0.3349650 .503201 & 0.302304 & 0.581701 \\
\hline
\end{tabular}

Table 7(b). Nano fluid thermal conductivity

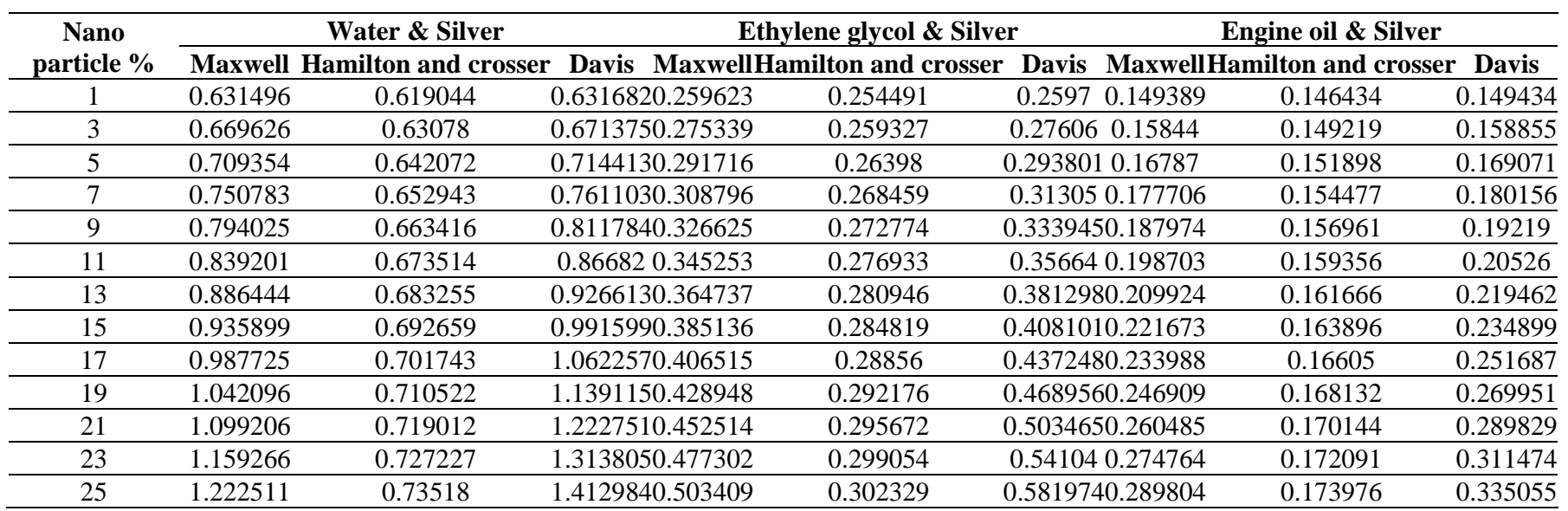

Table 7(b). Nano fluid thermal conductivity (continued)

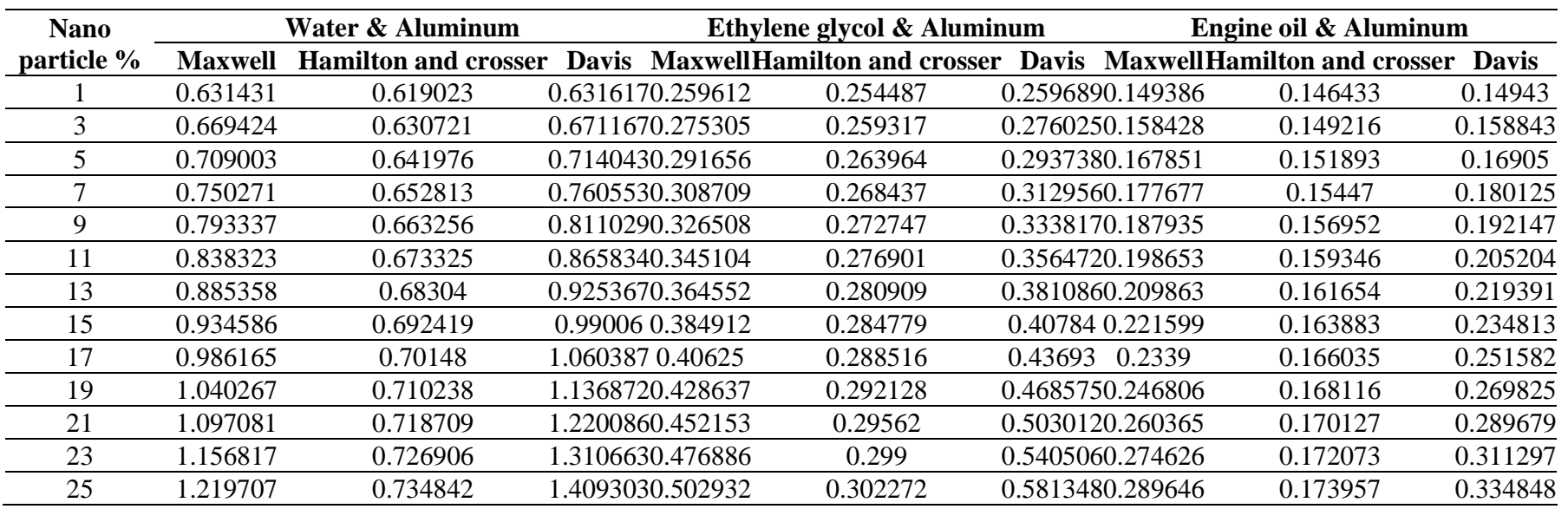

Further, the thermal conductivity of $1 \%$ particles with copper mix and water as base fluid is 0.63149 using Maxwell model, 0.619042 suing Hamilton and crosser model, and 0.631677 using Davis model. The thermal conductivity of different Nano particle mixtures for all the three models is given in Table 7(a) and Table 7(b).

From the results, it is found that the Maxwell model provides a better results with regular variation and the mean values has better values than the other two models. Since, most of the particles in the Nano fluid possess spherical in shape.

\section{RESULTS AND DISCUSSION}

This research considers the problem related to convection flow of Nano particles in a tube, which is heated uniformly. The chosen measurements for the study includes $6 \mathrm{~mm}$ diameter and length of $170 \mathrm{~mm}$ and the simulation analysis is carried out in Ansys Workbench tool.

The proposed tubular module is connected to a constant and uniform heat flux at the wall of the chamber. The analysis to find the heat transfer rate is analysed on base and Nano fluid with constant Reynolds number. 


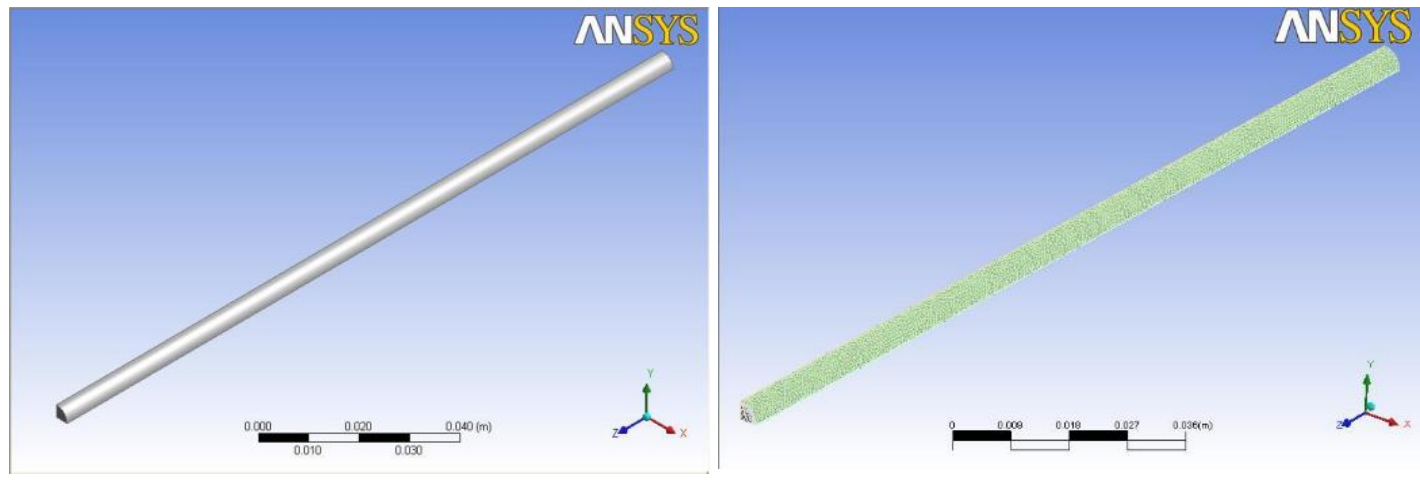

(a) Modeling after symmetry; (b) Modeling after meshing

Figure 3. 3D modelling of uniform heated tube
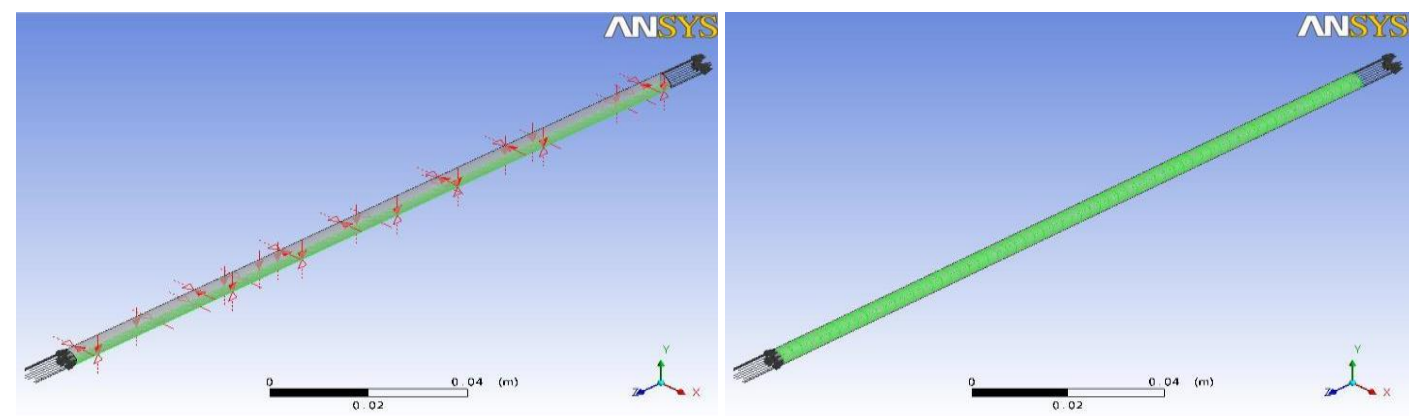

Figure 4. CFX Modelling

Table 8. Heat transfer co-efficientof Water, Ethylene glycol (E.GL) and Engine oil (E.Oil) with Nanoparticles at 333K

\begin{tabular}{|c|c|c|c|c|c|c|c|c|c|c|}
\hline \multirow{2}{*}{$\begin{array}{c}\text { Nano } \\
\text { particle } \%\end{array}$} & \multicolumn{2}{|c|}{$\mathrm{H}_{2} \mathrm{O}+\mathrm{Cu}$} & \multirow{2}{*}{$\begin{array}{l}\mathrm{H}_{2} \mathrm{O} \\
+\mathrm{Au}\end{array}$} & \multirow{2}{*}{$\begin{array}{r}\mathrm{H}_{2} \mathrm{O} \\
+\mathrm{Ag}\end{array}$} & \multicolumn{2}{|c|}{$\mathbf{H}_{2} \mathbf{O}+\mathbf{A l}$} & \multicolumn{2}{|c|}{ E.Gl + Cu } & \multirow{2}{*}{$\begin{array}{c}\text { E.Gl } \\
+\mathbf{A u}\end{array}$} & \multirow{2}{*}{$\begin{array}{l}\text { E.Gl } \\
+ \text { Ag }\end{array}$} \\
\hline & CFX & Experimental & & & CFX F & Experimental & CFX 1 & Experimental & & \\
\hline 1 & 13540 & 13689.4 & 13610.8 & 13553.11 & 13493.3 & 13520.36 & 3935.24 & 4018.2 & 3963.36 & 3940.53 \\
\hline 3 & 14533.5 & 14628.3 & 14681.2 & 14564.6 & 14412.6 & 14530.42 & 4262.92 & 4325.8 & \multicolumn{2}{|c|}{4351.454280 .27} \\
\hline 5 & \multicolumn{2}{|c|}{15542.4} & \multicolumn{2}{|c|}{15722.615584 .3} & \multicolumn{2}{|r|}{15367.1} & \multicolumn{2}{|c|}{4613.62} & 4766.56 & 4644.98 \\
\hline 7 & \multicolumn{2}{|c|}{16572.7} & \multicolumn{2}{|c|}{16762.816621} & & 6357.2 & \multicolumn{2}{|c|}{4985.12} & \multicolumn{2}{|c|}{5204.625032 .2} \\
\hline 9 & \multicolumn{2}{|c|}{17629.6} & \multicolumn{2}{|c|}{17816.817681 .4} & \multicolumn{2}{|r|}{17384.1} & \multicolumn{2}{|c|}{5376.37} & \multicolumn{2}{|c|}{5662.755440 .7} \\
\hline 11 & \multicolumn{2}{|c|}{18719} & \multicolumn{2}{|c|}{$\begin{array}{ll}18894 & 18772 \\
\end{array}$} & \multicolumn{2}{|r|}{18451.3} & \multicolumn{2}{|r|}{5788.12} & \multicolumn{2}{|c|}{6140.565870 .95} \\
\hline 13 & \multicolumn{2}{|c|}{19846.1} & \multicolumn{2}{|c|}{20003.919898 .5} & \multicolumn{2}{|r|}{19562.8} & \multicolumn{2}{|c|}{6222.22} & \multicolumn{2}{|c|}{6638.896324 .61} \\
\hline 15 & \multicolumn{2}{|c|}{21014.9} & \multicolumn{2}{|c|}{21159.921065 .5} & \multicolumn{2}{|r|}{20722.4} & \multicolumn{2}{|c|}{6680.16} & 7158.86 & 5802.98 \\
\hline 17 & & 2230.6 & 22366. & 22278 & & 21933.7 & & 7163.46 & 7701.48 & 7307.41 \\
\hline 19 & & 3497.6 & 23629.5 & 23543.2 & & 23200.6 & & 7673.6 & 8267.87 & 7839.16 \\
\hline 21 & & 4821.8 & 24951. & 24869.8 & & 24527 & & 3212.31 & 8859.32 & 3399.79 \\
\hline 23 & & 5213.4 & 26335. & 26261.3 & & 25918.9 & & 3782.36 & 9478.35 & 3991.85 \\
\hline 25 & & 7675.5 & 27784. & 27725.1 & & 27381.1 & & 385.15 & 10126.3 & 9616.54 \\
\hline
\end{tabular}

Table 8. Heat transfer co-efficientof Water, Ethylene glycol (E.GL) and Engine oil (E.Oil) with Nanoparticles at 333K (continued)

\begin{tabular}{|c|c|c|c|c|c|c|c|c|}
\hline \multirow{2}{*}{$\begin{array}{c}\text { Nano } \\
\text { particle } \%\end{array}$} & \multicolumn{2}{|c|}{ E.Gl + Al } & \multicolumn{2}{|c|}{ E.Oil+Cu } & \multirow{2}{*}{$\begin{array}{c}\text { E.Oil } \\
+\mathrm{Au} \\
\end{array}$} & \multirow{2}{*}{$\begin{array}{c}\text { E.Oil } \\
+ \text { Ag } \\
\end{array}$} & \multicolumn{2}{|c|}{ E.Oil +Al } \\
\hline & CFX & Experimental & CFX & Experimental & & & CFX & Experimental \\
\hline 1 & 3919.66 & 4022.3 & 2063.1 & 2098.13 & 2611.27 & 2063.21 & 2062.78 & 2125.35 \\
\hline 3 & 4212.63 & 4275.7 & 2203.46 & 2258.93 & 2685.83 & 2203.89 & 2202.32 & 2428.8 \\
\hline 5 & \multicolumn{2}{|c|}{4523.97} & \multicolumn{2}{|r|}{2351.2} & \multicolumn{2}{|c|}{2763.992352 .03} & \multicolumn{2}{|c|}{2349.1} \\
\hline 7 & \multicolumn{2}{|c|}{4851.74} & \multicolumn{2}{|c|}{2505.11} & \multicolumn{2}{|c|}{2843.932506 .48} & \multicolumn{2}{|r|}{2493.56} \\
\hline 9 & \multicolumn{2}{|c|}{5195.2} & \multicolumn{2}{|c|}{2664.68} & \multicolumn{2}{|c|}{2924.942666 .71} & \multicolumn{2}{|c|}{2659.89} \\
\hline 11 & \multicolumn{2}{|c|}{5555.59} & \multicolumn{2}{|c|}{2830.4} & \multicolumn{2}{|c|}{3007.392833 .25} & \multicolumn{2}{|r|}{2823.93} \\
\hline 13 & \multicolumn{2}{|c|}{5934.99} & \multicolumn{2}{|c|}{3003.09} & \multicolumn{2}{|c|}{3092.093006 .93} & \multicolumn{2}{|r|}{2994.63} \\
\hline 15 & \multicolumn{2}{|c|}{6335.11} & \multicolumn{2}{|c|}{3183.36} & \multicolumn{2}{|c|}{3179.353188 .41} & \multicolumn{2}{|c|}{3172.65} \\
\hline 17 & \multicolumn{2}{|c|}{6757.88} & \multicolumn{2}{|c|}{3372.03} & \multicolumn{2}{|c|}{3269.793378 .49} & \multicolumn{2}{|r|}{3358.75} \\
\hline 19 & \multicolumn{2}{|c|}{7205.07} & \multicolumn{2}{|c|}{3569.77} & \multicolumn{2}{|c|}{3363.773577 .91} & & 3553.57 \\
\hline 21 & & 578.88 & & 3777.6 & 3461.99 & 3787.74 & & 3758.06 \\
\hline 23 & & 82.34 & & 3996.7 & 3565.12 & 4009.16 & & 3973.32 \\
\hline 25 & & 717.26 & & 229.73 & 3673.56 & 4243.13 & & 4200.22 \\
\hline
\end{tabular}




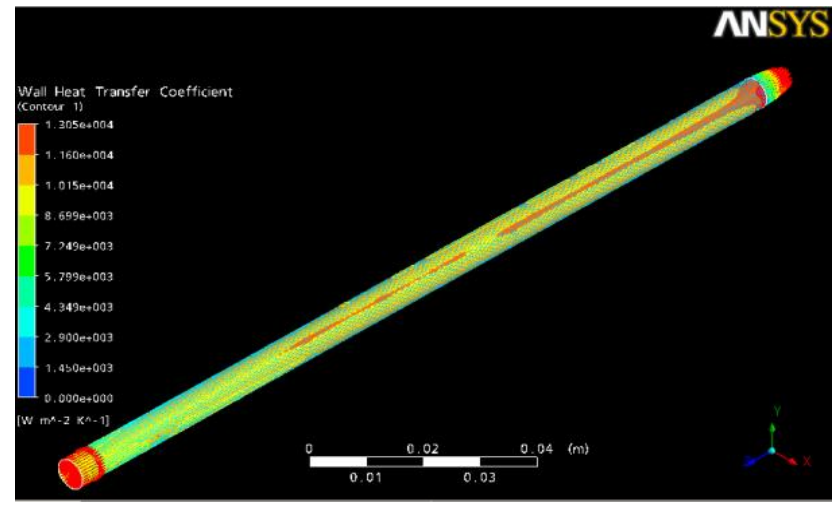

Figure 5. Heat transfer co-efficient of water at $333 \mathrm{~K}$

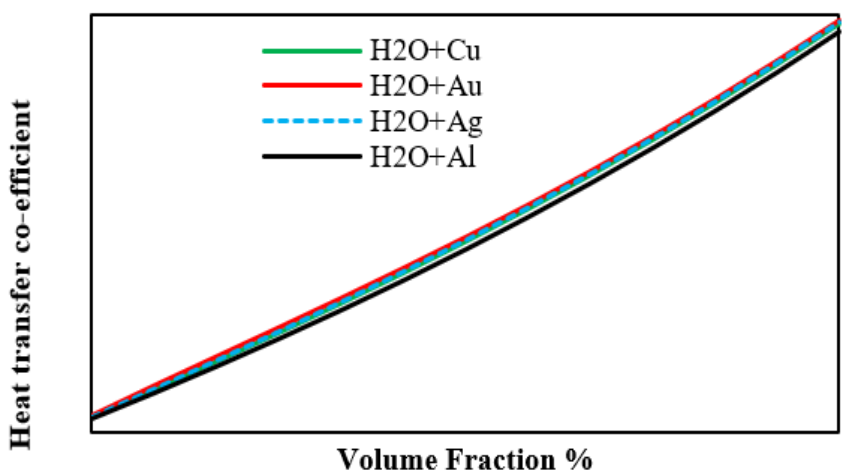

Figure 6. Heat transfer co-efficient of Water with Nano particles at $333 \mathrm{~K}$

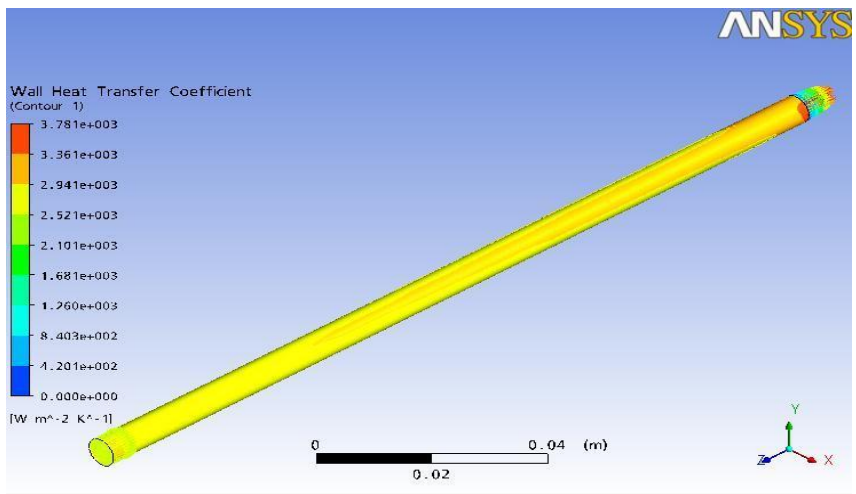

Figure 7. Heat transfer co-efficient of ethyleneglycolat $333 \mathrm{~K}$

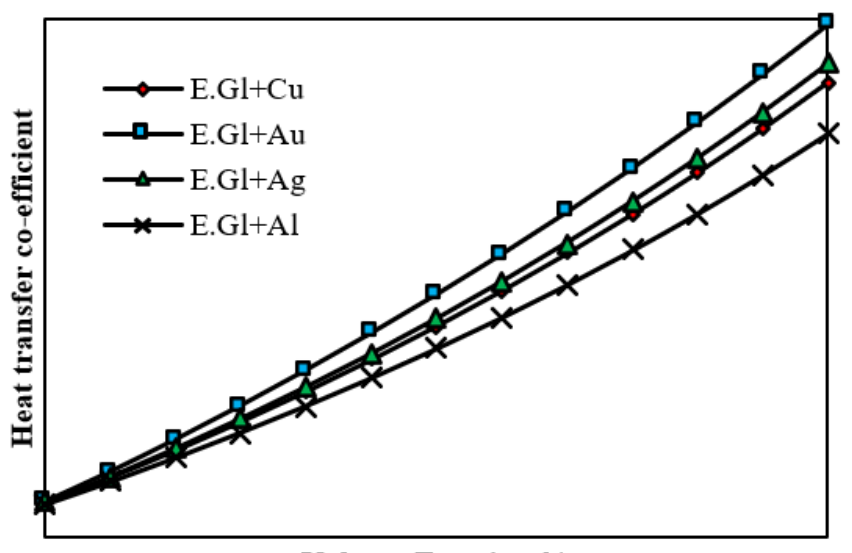

Volume Fraction \%

Figure 8. Heat transfer co-efficient of Ethylene glycol with Nano particles at $333 \mathrm{~K}$

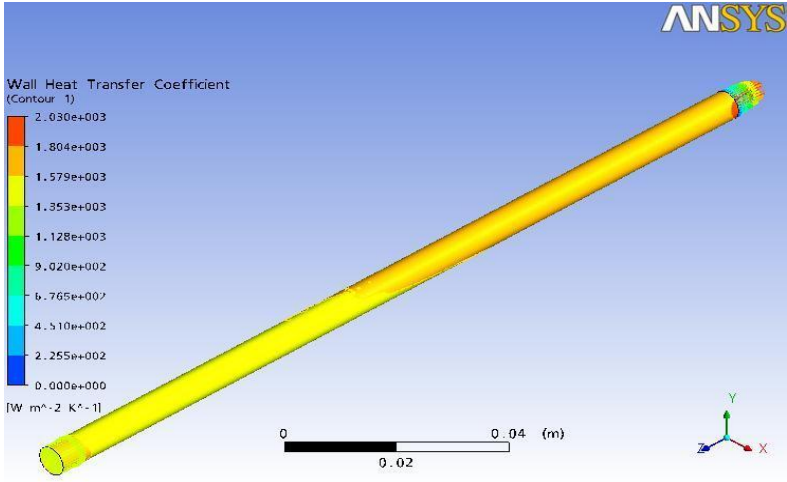

Figure 9. Heat transfer co-efficient of engineoil at 333K

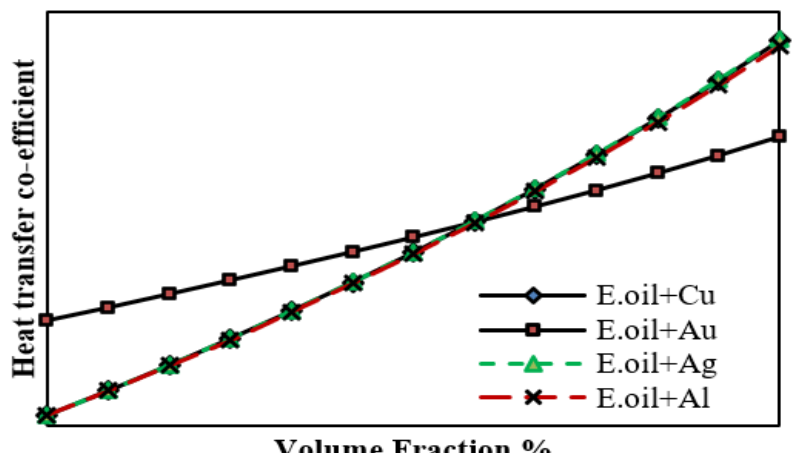

Figure 10. Heat transfer co-efficient of Engineoil with Nanoparticles at $333 \mathrm{~K}$

The analytical work is carried out with a CFX11.0 module inside the tubular structure, as shown in Figure 4. The modeling is done in Ansys Workbench 11.0 and imported into CFX11.0 software to analyse the flow and finding the heat transfer co-efficient of water.

From the analysis, it is found that the heat transfer coefficient (Figure 5) for water is $13048.1 \mathrm{~W} / \mathrm{m}^{2} \mathrm{~K}$ at $333 \mathrm{~K}$. From Table 7 or Figure 6, it is seen that the Nano fluids other than copper, gold, silver and aluminum Nanoparticles mixture with water proves a higher heat transfer rate than water in both experimental and simulation method. Likewise, it is found that the heat transfer coefficient (Figure 7) for ethylene glycol is $3781.16 \mathrm{~W} / \mathrm{m}^{2} \mathrm{~K}$ at $333 \mathrm{~K}$.

From Table 8 or Figure 8 , it is seen that the Nano fluids other than copper, gold, silver and aluminum Nanoparticles mixture with ethylene glycol proves a higher heat transfer rate than ethylene glycol in both experimental and simulation method. Also, it is found that the heat transfer coefficient (Figure 9) for engine oil is $1996.36 \mathrm{~W} / \mathrm{m}^{2} \mathrm{~K}$ at $333 \mathrm{~K}$. From Table 7 or Figure 10, it is seen that the Nano fluids other than copper, gold, silver and aluminum Nanoparticles mixture with ethylene glycol proves a higher heat transfer rate than engine oil in both experimental and simulation method.

\section{CONCLUSIONS}

This paper solves the convective heat transfer problem using a heated tube at $333 \mathrm{~K}$ over base and Nano fluids and that includes: water, ethylene glycol and engine oilas base fluids and Water/ Nano particles of Copper, Gold, Silver and Aluminum, Ethyleneglycol/ Nanoparticles of Copper, Gold, Silver and Aluminum, Engineoil/ Nanoparticles of Copper, 
Gold, Silver and Aluminum as Nano fluid combination. The performance results prove that the Nano fluids possess higher heat transfer coefficient than the base fluid under constant Reynolds number. The Nanoparticles suspended in the base fluid increases constantly the performance of convective heat transfer rate and it could further be concluded that with constant Reynolds number, the heat transfer coefficient has increased with its associated particle volume fraction

\section{REFERENCES}

[1] Choi, S.U., Eastman, J.A. (1995). Enhancing thermal conductivity of fluids with nanoparticles (No. ANL/MSD/CP-84938; CONF-951135-29). Argonne National Lab., IL (United States). 99-106.

[2] Yang, C., Wu, X., Zheng, Y., Qiu, T. (2017). Heat transfer performance assessment of hybrid nanofluids in a parallel channel under identical pumping power. Chemical Engineering Science, 168: 67-77. https://doi.org/10.1016/j.ces.2017.04.045

[3] Gupta, M., Singh, V., Kumar, R., Said, Z. (2017). A review on thermophysical properties of nanofluids and heat transfer applications. Renewable and Sustainable Energy Reviews, 74: 638-670. https://doi.org/10.1016/j.rser.2017.02.073

[4] Akoh, H., Tsukasaki, Y., Yatsuya, S., Tasaki, A. (1978). Magnetic properties of ferromagnetic ultrafine particles prepared by vacuum evaporation on running oil substrate. Journal of Crystal Growth, 45: 495-500. https://doi.org/10.1016/0022-0248(78)90482-7

[5] Liu, K.V., Choi, U.S., Kasza, K.E. (1988). Measurements of pressure drop and heat transfer in turbulent pipe flows of particulate slurries. STIN, 89: 11150.

[6] Chen, L., Xie, H. (2009). Silicon oil based multiwalled carbon nanotubes nanofluid with optimized thermal conductivity enhancement. Colloids and Surfaces A: Physicochemical and Engineering Aspects, 352(1-3): 136-140. https://doi.org/10.1016/j.colsurfa.2009.10.015

[7] Wei, X., Zhu, H., Kong, T., Wang, L. (2009). Synthesis and thermal conductivity of $\mathrm{Cu}_{2} \mathrm{O}$ nanofluids. International Journal of Heat and Mass Transfer, 52(1920):

4371-4374 https://doi.org/10.1016/j.ijheatmasstransfer.2009.03.073

[8] Yu, W., Xie, H., Chen, L., Li, Y. (2010). Enhancement of thermal conductivity of kerosene-based $\mathrm{Fe}_{3} \mathrm{O}_{4}$ nanofluids prepared via phase-transfer method. Colloids and surfaces A: Physicochemical and engineering aspects, 355(1-3): 109-113. https://doi.org/10.1016/j.colsurfa.2009.11.044

[9] Murshed, S.M.S., Leong, K.C., Yang, C. (2005). Enhanced thermal conductivity of $\mathrm{TiO}_{2}$-water based nanofluids. International Journal of thermal sciences, 44(4):

$367-373$ https://doi.org/10.1016/j.ijthermalsci.2004.12.005

[10] Buongiorno, J., Venerus, D.C., Prabhat, N., McKrell, T., Townsend, J., Christianson, R., Bang, I.C. (2009). A benchmark study on the thermal conductivity of nanofluids. Journal of Applied Physics, 106(9): 094312.

[11] Zhu, D., Li, X., Wang, N., Wang, X., Gao, J., Li, H. (2009). Dispersion behavior and thermal conductivity characteristics of $\mathrm{Al}_{2} \mathrm{O}_{3}-\mathrm{H}_{2} \mathrm{O}$ nanofluids. Current
Applied Physics,
https://doi.org/10.1016/j.cap.2007.12.008

131-139.

[12] Teng, T.P., Hung, Y.H., Teng, T.C., Mo, H.E., Hsu, H.G. (2010). The effect of alumina/water nanofluid particle size on thermal conductivity. Applied Thermal Engineering, 30(14-15): 2213-2218. https://doi.org/10.1016/j.applthermaleng.2010.05.036

[13] Sundar, L.S., Singh, M.K., Sousa, A.C. (2013). Investigation of thermal conductivity and viscosity of $\mathrm{Fe}_{3} \mathrm{O}_{4}$ nanofluid for heat transfer applications. International communications in heat and mass transfer, 44:

7-14. https://doi.org/10.1016/j.icheatmasstransfer.2013.02.01 4

[14] Cuenca, Y., Salavera, D., Vernet, A., Vallès, M. (2013). Thermal conductivity of ammonia+water mixtures over a wide range of concentrations. International Journal of Refrigeration, 36(3): 998-1003. https://doi.org/10.1016/j.ijrefrig.2012.10.039

[15] Said, Z., Saidur, R., Hepbasli, A., Rahim, N.A. (2014). New thermophysical properties of water based $\mathrm{TiO}_{2}$ nanofluid-The hysteresis phenomenon revisited. International Communications in Heat and Mass Transfer, 58: 85-95. https://doi.org/10.1016/j.icheatmasstransfer.2014.08.03 4

[16] Esfe, M.H., Saedodin, S., Mahmoodi, M. (2014). Experimental studies on the convective heat transfer performance and thermophysical properties of $\mathrm{MgO}$ water nanofluid under turbulent flow. Experimental thermal and fluid science, 52: 68-78. https://doi.org/10.1016/j.expthermflusci.2013.08.023

[17] Esfe, M.H., Saedodin, S., Mahian, O., Wongwises, S. (2014). Thermophysical properties, heat transfer and pressure drop of $\mathrm{COOH}$-functionalized multi walled carbon nanotubes/water nanofluids. International Communications in Heat and Mass Transfer, 58: 176-183. https://doi.org/10.1016/j.icheatmasstransfer.2014.08.03 7

[18] Li, X.F., Zhu, D.S., Wang, X.J., Wang, N., Gao, J.W., Li, H. (2008). Thermal conductivity enhancement dependent $\mathrm{pH}$ and chemical surfactant for $\mathrm{Cu}-\mathrm{H}_{2} \mathrm{O}$ nanofluids. Thermochimica Acta, 469(1-2): 98-103. https://doi.org/10.1016/j.tca.2008.01.008

[19] Megatif, L., Ghozatloo, A., Arimi, A., Shariati-Niasar, M. (2016). Investigation of laminar convective heat transfer of a novel $\mathrm{TiO}_{2}$-carbon nanotube hybrid water-based nanofluid. Experimental Heat Transfer, 29(1): 124-138.

[20] Nasiri, A., Shariaty-Niasar, M., Rashidi, A.M., Khodafarin, R. (2012). Effect of CNT structures on thermal conductivity and stability of nanofluid. International Journal of Heat and Mass Transfer, 55(5-6): $1529-1535$

https://doi.org/10.1016/j.ijheatmasstransfer.2011.11.004

[21] Yeganeh, M., Shahtahmasebi, N., Kompany, A.H.M.A.D., Goharshadi, E.K., Youssefi, A., Šiller, L. (2010). Volume fraction and temperature variations of the effective thermal conductivity of nanodiamond fluids in deionized water. International Journal of Heat and Mass Transfer, 53(15-16): 3186-3192. https://doi.org/10.1016/j.ijheatmasstransfer.2010.03.008

[22] Gandhi, K.S.K., Velayutham, M., Das, S.K., Thirumalachari, S. (2011). Measurement of thermal and electrical conductivities of graphene nanofluids. 
[23] Jiang, H., Li, H., Xu, Q., Shi, L. (2014). Effective thermal conductivity of nanofluids Considering interfacial nanoshells. Materials Chemistry and Physics, 148(1-2): 195200.

https://doi.org/10.1016/j.matchemphys.2014.07.031

[24] Hajjar, Z., morad Rashidi, A., Ghozatloo, A. (2014). Enhanced thermal conductivities of graphene oxide nanofluids. International Communications in Heat and Mass Transfer, 57: 128-131. https://doi.org/10.1016/j.icheatmasstransfer.2014.07.01 8

[25] Sundar, L.S., Hortiguela, M.J., Singh, M.K., Sousa, A.C. (2016). Thermal conductivity and viscosity of water based nanodiamond (ND) nanofluids: An experimental study. International Communications in Heat and Mass Transfer, 76: 245-255. https://doi.org/10.1016/j.icheatmasstransfer.2016.05.02 5

[26] Liu, M.S., Lin, M.C.C., Huang, I.T., Wang, C.C. (2005). Enhancement of thermal conductivity with carbon nanotube for nanofluids. International Communications in Heat and Mass Transfer, 32(9): 1202-1210. https://doi.org/10.1016/j.icheatmasstransfer.2005.05.00 5

[27] Yu, W., Xie, H., Chen, L., Li, Y. (2009). Investigation of thermal conductivity and viscosity of ethylene glycol based $\mathrm{ZnO}$ nanofluid. Thermochimica Acta, 491(1-2): 92-96.

[28] Moosavi, M., Goharshadi, E.K., Youssefi, A. (2010). Fabrication, characterization, and measurement of some physicochemical properties of $\mathrm{ZnO}$ nanofluids. International Journal of Heat and Fluid Flow, 31(4): 599 605.

https://doi.org/10.1016/j.ijheatfluidflow.2010.01.011

[29] Paul, G., Philip, J., Raj, B., Das, P.K., Manna, I. (2011). Synthesis, characterization, and thermal property measurement of nano-A195Zn05 dispersed nanofluid prepared by a two-step process. International Journal of Heat and Mass Transfer, 54(15-16): 3783-3788. https://doi.org/10.1016/j.ijheatmasstransfer.2011.02.044

[30] Yu, W., Xie, H., Wang, X., Wang, X. (2011). Significant thermal conductivity enhancement for nanofluids containing graphene nanosheets. Physics Letters A, 375(10):

1323-1328 https://doi.org/10.1016/j.physleta.2011.01.040

[31] Yu, W., Xie, H., Li, Y., Chen, L. (2011). Experimental investigation on thermal conductivity and viscosity of aluminum nitride nanofluid. Particuology, 9(2): 187-191. https://doi.org/10.1016/j.partic.2010.05.014

[32] Chopkar, M., Kumar, S., Bhandari, D.R., Das, P.K., Manna, I. (2007). Development and characterization of $\mathrm{Al}_{2} \mathrm{Cu}$ and $\mathrm{Ag}_{2} \mathrm{Al}$ nanoparticle dispersed water and ethylene glycol based nanofluid. Materials Science and Engineering: $\quad \mathrm{B}, \quad$ 139(2-3): 141-148. https://doi.org/10.1016/j.mseb.2007.01.048

[33] Vajjha, R.S., Das, D.K. (2009). Experimental determination of thermal conductivity of three nanofluids and development of new correlations. International Journal of Heat and Mass Transfer, 52(21-22): $4675-$ 4682

https://doi.org/10.1016/j.ijheatmasstransfer.2009.06.027
[34] Reddy, M.C.S., Rao, V.V. (2013). Experimental studies on thermal conductivity of blends of ethylene glycolwater-based $\mathrm{TiO}_{2}$ nanofluids. International Communications in Heat and Mass Transfer, 46: 31-36. https://doi.org/10.1016/j.icheatmasstransfer.2013.05.00 9

[35] Sahooli, M., Sabbaghi, S. (2013). Investigation of thermal properties of $\mathrm{CuO}$ nanoparticles on the ethylene glycol-water mixture. Materials Letters, 93: 254-257. https://doi.org/10.1016/j.matlet.2012.11.097

[36] Nikkam, N., Ghanbarpour, M., Saleemi, M., Haghighi, E. B., Khodabandeh, R., Muhammed, M., Toprak, M.S. (2014). Experimental investigation on thermo-physical properties of copper/diethylene glycol nanofluids fabricated via microwave-assisted route. Applied Thermal Engineering, 65(1-2): 158-165. https://doi.org/10.1016/j.applthermaleng.2014.01.003

[37] Nikkam, N., Haghighi, E.B., Saleemi, M., Behi, M., Khodabandeh, R., Muhammed, M., Toprak, M.S. (2014). Experimental study on preparation and base liquid effect on thermo-physical and heat transport characteristics of $\alpha-\mathrm{SiC}$ nanofluids. International Communications in Heat and Mass Transfer, 55: 38-44. https://doi.org/10.1016/j.icheatmasstransfer.2014.04.01 1

[38] Suganthi, K.S., Vinodhan, V.L., Rajan, K.S. (2014). Heat transfer performance and transport properties of $\mathrm{ZnO}$ ethylene glycol and $\mathrm{ZnO}$-ethylene glycol-water nanofluid coolants. Applied energy, 135: 548-559. https://doi.org/10.1016/j.apenergy.2014.09.023

[39] Elias, M.M., Mahbubul, I.M., Saidur, R., Sohel, M.R., Shahrul, I.M., Khaleduzzaman, S.S., Sadeghipour, S. (2014). Experimental investigation on the thermophysical properties of $\mathrm{Al}_{2} \mathrm{O}_{3}$ nanoparticles suspended in car radiator coolant. International Communications in Heat and Mass Transfer, 54: 48-53. https://doi.org/10.1016/j.icheatmasstransfer.2014.03.00 5

[40] Sundar, L.S., Ramana, E.V., Singh, M.K., Sousa, A.C. (2014). Thermal conductivity and viscosity of stabilized ethylene glycol and water mixture $\mathrm{Al}_{2} \mathrm{O}_{3}$ nanofluids for heat transfer applications: An experimental study. International Communications in Heat and Mass Transfer, 56: 86-95. https://doi.org/10.1016/j.icheatmasstransfer.2014.06.00 9

[41] Sathish, T. (2017). Performance measurement on extracted bio-diesel from waste plastic. Journal of Applied Fluid Mechanics, 10: 41-50.

[42] Sathish, T. (2017). Heat Transfer Analysis Of Nano-fluid Flow In A Converging Nozzle With Different Aspect Ratios. Journal of New Materials for Electrochemical Systems, 20(4): 161-167. https://doi.org/10.14447/jnmes.v20i4.321

\section{NOMENCLATURES}

$\begin{array}{lll}v_{i} & {\left[\mathrm{~ms}^{-1}\right]} & \text { velocity in } i^{\text {th }} \text { position } \\ t_{\max } & {[\mathrm{min}]} & \text { maximal time limit } \\ T_{0} & {[\mathrm{~K}]} & \text { initial temperature }\end{array}$

